\title{
Behaviour of the intensity current in a membrane covered disc microelectrode under steady-state conditions
}

\author{
Josep Galceran $^{\mathrm{a} *}$, José Salvador ${ }^{\mathrm{a}}$, Jaume Puy ${ }^{\mathrm{a}}$, Joan Cecília ${ }^{\mathrm{b}}$ and David Gavaghan ${ }^{\mathrm{c}}$ \\ ${ }^{a}$ Dept. Química, ETSEA, Universitat de Lleida, Rovira Roure 177, 25198, Lleida, Spain \\ ${ }^{\mathrm{b}}$ Dept. Matemàtica, ETSEA, Universitat de Lleida, Rovira Roure 177, 25198, Lleida, Spain \\ ${ }^{\mathrm{c} O x f o r d}$ University Computing Laboratory, Wolfson Building, Parks Road, Oxford OX1 3QD, \\ UK and Nuffield Department of Anaesthetics, Radcliffe Infirmary, Oxford, UK \\ * corresponding author
}

\section{Abstract}

The inlaid disc electrode covered with a membrane can be modelled as a system with axial symmetry containing several parallel layers with different permeabilities. A recent solution of the steady state under diffusion limited conditions of an electroactive species in such a system is implemented with Mathematica and the code for the exact solution and an approximation is provided. Computation of the theoretical intensity current becomes an easy and fast procedure, capable of being implemented on any personal computer or workstation. The effect of the membrane permeability, the thickness of the membrane, the electrode radius and the electrolyte layer thickness on the intensity current are analysed. We define an index of edgeeffect contribution of each layer to the overall flux, providing their formulae together with the physical interpretation. For many sets of parameters, the edge-effect is responsible for an important contribution of the radial diffusion within the electrolyte and membrane. More accurate determination of the permeabilities of the different media is now possible experimentally, as the functional dependence of the current on the physical parameters is readily available for fitting procedures.

Keywords: steady state, disk electrode, permeability, membrane covered microelectrode

\section{Introduction}

A wide range of electrochemical sensors, such as the ion selective electrodes or the Clark oxygen sensor, rely on the characteristics of a membrane that splits the original sample medium into a multilayered one. The protective membrane separates the sample medium from an electrolyte solution close to the electrode ${ }^{1-3}$. Several modes of operation are available, but steady state measurements under diffusion limited conditions are amongst the most frequent in the experimental use of the inlaid covered electrode ${ }^{4}$.

Mathematically, the steady-state inlaid electrode system in a multilayered medium had been previously modelled using a one-dimensional approach ${ }^{5-7}$ (unable to account for the "edgeeffect" ${ }^{8-10}$ ) or a "mixed" (radial/axial) approach". All such attempts are restricted to gas sample or thoroughly stirred liquid sample sensors. Electrochemical permeability determinations have been carried out through various methods usually relying on onedimensional models ${ }^{12-15}$.

In the "axisymmetric" or "cylindrical" model, 3 horizontal layers (electrolyte, membrane and sample solution) extend up to infinity parallel to the disk and the coplanar insulator around it 
$11,16,17$. Numerical simulation of the currents either through finite differences or finite elements ${ }^{18,19}$ involve considerable computational effort.

Recently ${ }^{20}$, a rigorous method has been presented for solving for the diffusive flux of an electroactive species towards an inlaid disc electrode in the steady state under diffusion limited conditions. By reformulating the problem as a dual integral equation, the unknowns are just the coefficients of an infinite series expansion ${ }^{21-23}$. Using this new analytical solution we have been able to demonstrate the restricted range of applicability ${ }^{20}$ of previous (approximate) models. In principle, the new formulation applies to any disk electrode covered with any number of infinite (in the radial direction) layers of different media, although practical reasons (interference of convection or the length of time needed to reach the steady state when using large electrodes) may restrict its applicability to small electrode radii. The ease of implementation (on a personal computer or on a workstation with modern software) of the results there found allow us to perform here further studies such as the influence of the physical parameters on the intensity current or the determination of the media permeabilities.

More specifically, in this work, we aim: i) to implement this rigorous method of solution providing the code in Mathematica ${ }^{24}$ ); ii) to characterise the contribution of each layer to the edge effect by introducing new indices and computing them; iii) to point out several analytical approximations (not restricted to gas sensors or stirred liquid samples) which allow procedures such as the fast determination of the permeabilities of the different layers from intensity current measurements; iv) to study the effect of those physical parameters which have greatest influence on the current.

\section{Axisymmetric model}

The electrode can be considered as a disc, of radius $r_{c}$, embedded in and coplanar with an insulator extending out to infinity. Figure 1 shows a two-dimensional section of the modelled system. The line perpendicular to the disc through its centre defines the z-axis, which is also a symmetry axis of the whole system. The electrode radius is taken as the unity of length, so that all other distances are normalised with respect to it. Three layers (electrolyte, membrane and sample) extend parallel to the insulator surface, with their boundaries at distances $\mathrm{z}_{\mathrm{e}}$ and $\mathrm{Z}_{\mathrm{m}}$. We caution that $\mathrm{Z}_{\mathrm{m}}$ is the normalised distance between the electrode-insulator plane and the upper membrane surface, and not the thickness of the membrane layer. Each layer represents a different medium in which the electroactive species diffuses differently according to its permeability $\mathrm{P}_{\mathrm{e}}, \mathrm{P}_{\mathrm{m}}$ or $\mathrm{P}_{\mathrm{s}}$. As useful combinations of the permeabilities we introduce the non-dimensional parameters:

$\varepsilon_{1} \equiv \frac{\mathrm{P}_{\mathrm{e}}-\mathrm{P}_{\mathrm{m}}}{\mathrm{P}_{\mathrm{e}}+\mathrm{P}_{\mathrm{m}}} \quad \varepsilon_{2} \equiv \frac{\mathrm{P}_{\mathrm{s}}-\mathrm{P}_{\mathrm{m}}}{\mathrm{P}_{\mathrm{s}}+\mathrm{P}_{\mathrm{m}}}$

In Appendix A we detail the differential equation corresponding to the steady state and the boundary conditions of the diffusion limited current.

\section{Evaluation of the non-dimensional intensity current}

The steady state intensity current under diffusion limited conditions towards an unshielded inlaid disc electrode in an electrolyte medium is well known ${ }^{25,26}$ : 
$\mathrm{i}_{\mathrm{d}, \mathrm{e}}=4 n \mathrm{FP}_{\mathrm{e}} \mathrm{p}^{*} \mathrm{r}=4 n \mathrm{FD}_{\mathrm{e}} \mathrm{c}^{*} \mathrm{r}$

where $n$ is the number of exchanged electrons, $\mathrm{F}$ the Faraday constant, $\mathrm{P}_{\mathrm{e}}$ the electrolyte permeability, $D_{e}$ the electrolyte diffusion coefficient and $\mathrm{c}^{*}$ the bulk electroactive concentration. We define the non-dimensional current, easily available from experiments, as the ratio of the steady state intensity current of the covered electrode to the current in the unshielded electrode (2). It has been shown ${ }^{20}$ that the steady state of a disk electrode covered with multilayered media may be reformulated in terms of a dual integral equation and that the non-dimensional current can be computed through an exceedingly simple expression:

$\frac{\mathrm{i}}{\mathrm{i}_{\mathrm{d}, \mathrm{e}}}=\sqrt{\frac{\pi}{2}} \mathrm{a}_{0}$

where $\mathrm{a}_{0}$ is found by truncating and solving the infinite system of linear equations ${ }^{21}$ :

$$
\begin{array}{ll}
\mathrm{a}_{0}+\sum_{\mathrm{m}=0}^{\infty} \mathrm{L}_{\mathrm{m}, 0} \mathrm{a}_{\mathrm{m}}=\sqrt{\frac{2}{\pi}} & \\
\mathrm{a}_{\mathrm{n}}+\sum_{\mathrm{m}=0}^{\infty} \mathrm{L}_{\mathrm{m}, \mathrm{n}} \mathrm{a}_{\mathrm{m}}=0 & (\mathrm{n}>0)
\end{array}
$$

where

$$
\mathrm{L}_{\mathrm{m}, \mathrm{n}} \equiv(4 \mathrm{n}+1) \int_{0}^{\infty} \frac{2}{\lambda} \mathrm{f}_{3}(\lambda) \mathrm{J}_{2 \mathrm{~m}+1 / 2}(\lambda) \mathrm{J}_{2 \mathrm{n}+1 / 2}(\lambda) \mathrm{d} \lambda
$$

and $\mathrm{J}_{\mathrm{v}}$ stands for the Bessel function of order $v$ and

$$
\mathrm{f}_{3}(\lambda) \equiv \frac{\varepsilon_{1} \mathrm{e}^{-2 \lambda z_{\mathrm{e}}}-\varepsilon_{2} \mathrm{e}^{-2 \lambda z_{\mathrm{m}}}}{1-\varepsilon_{1} \varepsilon_{2} \mathrm{e}^{2 \lambda\left(\mathrm{z}_{\mathrm{e}}-z_{\mathrm{m}}\right)}-\varepsilon_{1} \mathrm{e}^{-2 \lambda z_{\mathrm{e}}}+\varepsilon_{2} \mathrm{e}^{-2 \lambda z_{\mathrm{m}}}} .
$$

In subsection a) of Appendix B there is an example of a Mathematica code to perform all of the operations needed in computing the non-dimensional intensity current for a standard case.

\section{Index of edge effect contribution}

Bond et al. ${ }^{27}$ proposed an "heterogeneity index" to characterise the distribution of the current at the unshielded disc surface, where the lack of uniformity of the flux density can be ascribed to the edge effect. Cope and Tallman ${ }^{28}$ also proposed an "edge effect coefficient" in the time dependent intensity current of a general (unshielded) planar electrode. In a covered electrode, we are concerned with the impact of each layer thickness and permeability in the spatial distribution of the flux of the electroactive species. In order to somehow characterise the edge effect contribution of each layer to the overall flux arriving at the electrode surface, we introduce the "edge effect index" $\eta_{\ell}$ of a layer $\ell$ as the fraction of this overall flux that has crossed the cylindrical surface $r=r_{c}$, within the layer $\ell$,

$$
\eta_{\iota} \equiv \frac{\int_{\ell} \mathrm{P}_{\iota}\left(\frac{\partial \mathrm{u}}{\partial \mathrm{r}}\right)_{\mathrm{r}=\mathrm{r}_{\mathrm{c}}} 2 \pi \mathrm{r}_{\mathrm{c}} \mathrm{dz}}{\int_{0}^{\mathrm{r}_{\mathrm{c}}} \mathrm{P}_{\mathrm{e}}\left(\frac{\partial \mathrm{u}}{\partial \mathrm{z}}\right)_{\mathrm{z}=0} 2 \pi \mathrm{rdr}}
$$


If we apply this definition to the particular case of an unshielded disc electrode (one medium), the cylindrical surface extends up to infinity, where its top circle is crossed by a negligible flux (this is a property of the steady state ${ }^{29}$ under semi-infinite diffusion: an infinite surface crossed by a infinitesimal density flux yielding a finite flux). So, we can conclude that the edge effect index for the electrolyte $\left(\eta_{\mathrm{e}}\right)$ is 1 , because in the steady state with no source, the flux entering a closed surface (e.g. through the cylindrical lateral surface) must be equal to the exiting flux (e.g. across the electrode surface).

If we apply the flux balance to the usual 3 layers, where the upper medium extends up to infinity with bulk boundary conditions at $\mathrm{z}=\infty$, we obtain

$\eta_{\mathrm{e}}+\eta_{\mathrm{m}}+\eta_{\mathrm{s}}=1$

After some algebra, by calculating the flux crossing the surface $\mathrm{z}=\mathrm{z}_{\mathrm{s}}$, between $\mathrm{r}=0$ and $\mathrm{r}=\mathrm{r}_{\mathrm{c}}$, it can be seen that

$\eta_{\mathrm{s}}=\left(\frac{1-\varepsilon_{1}}{1+\varepsilon_{1}}\right) \sqrt{\frac{\pi}{2}} \sum_{\mathrm{n}=0}^{\infty} \frac{\mathrm{a}_{\mathrm{n}}}{\mathrm{a}_{0}} \int_{0}^{\infty} \frac{\mathrm{J}_{2 \mathrm{n}+1 / 2}(\lambda) \mathrm{J}_{1}(\lambda) \mathrm{h}_{\mathrm{m}}(\lambda)}{\sqrt{\lambda}} \mathrm{d} \lambda$

where

$\mathrm{h}_{\mathrm{m}}(\lambda)=\frac{\mathrm{e}^{-\lambda z_{\mathrm{c}}}\left(1-\varepsilon_{1} \varepsilon_{2} \mathrm{e}^{2 \lambda\left(\mathrm{z}_{\mathrm{e}}-z_{\mathrm{m}}\right)}-\varepsilon_{1}+\varepsilon_{2} \mathrm{e}^{2 \lambda\left(\mathrm{z}_{\mathrm{e}}-z_{\mathrm{m}}\right)}\right)\left(1+\varepsilon_{2}\right)}{\left(1-\varepsilon_{1} \varepsilon_{2} \mathrm{e}^{2 \lambda\left(\mathrm{z}_{\mathrm{e}}-z_{\mathrm{m}}\right)}-\varepsilon_{1} \mathrm{e}^{-2 \lambda z_{\mathrm{e}}}+\varepsilon_{2} \mathrm{e}^{-2 \lambda z_{\mathrm{m}}}\right)\left(1-\varepsilon_{2} \mathrm{e}^{2 \lambda\left(\mathrm{z}_{\mathrm{e}}-z_{\mathrm{m}}\right)}\right)}$

Analogously, by calculating the flux crossing the surface $\mathrm{z}=\mathrm{z}_{\mathrm{e}}$, between $\mathrm{r}=0$ and $\mathrm{r}=\mathrm{r}_{\mathrm{c}}$, we obtain

$\eta_{\mathrm{s}}+\eta_{\mathrm{m}}=\sqrt{\frac{\pi}{2}} \sum_{\mathrm{n}=0}^{\infty} \frac{\mathrm{a}_{\mathrm{n}}}{\mathrm{a}_{0}} \int_{0}^{\infty} \frac{\mathrm{J}_{2 \mathrm{n}+1 / 2}(\lambda) \mathrm{J}_{1}(\lambda) \mathrm{h}_{\mathrm{e}}(\lambda)}{\sqrt{\lambda}} \mathrm{d} \lambda$

where

$h_{e}(\lambda)=\frac{\mathrm{e}^{-\lambda z_{\mathrm{e}}}\left(1-\varepsilon_{1} \varepsilon_{2} \mathrm{e}^{2 \lambda\left(z_{\mathrm{e}}-z_{\mathrm{m}}\right)}-\varepsilon_{1}+\varepsilon_{2} \mathrm{e}^{2 \lambda\left(z_{\mathrm{e}}-z_{\mathrm{m}}\right)}\right)}{1-\varepsilon_{1} \varepsilon_{2} \mathrm{e}^{2 \lambda\left(\mathrm{z}_{\mathrm{e}}-z_{\mathrm{m}}\right)}-\varepsilon_{1} \mathrm{e}^{-2 \lambda z_{\mathrm{e}}}+\varepsilon_{2} \mathrm{e}^{-2 \lambda z_{\mathrm{m}}}}$

For instance, with the data of figure 1 , it is found $\eta_{\mathrm{s}}=0.0860, \eta_{\mathrm{m}}=0.0108, \eta_{\mathrm{e}}=0.9032$. These values give an idea of the behaviour of the gradient lines, without the need of plotting them. Thus, it could be foreseen that just less than $10 \%$ of the flux crosses the sample-membrane interface above the electrode and that the radial diffusion in the electrolyte layer is dominant. It is seen in figure 1 that in a distance of 3 times the electrode radius just $40 \%$ of the incoming flux has crossed the membrane. Thus, it must be understood that $\eta_{\mathrm{s}}=0.0860$ does not mean that around $92 \%$ of the flux comes from the electrolyte and membrane layers, as flux crosses from the sample to the membrane all along their interface; but it does mean that the radial diffusion within both the electrolyte and membrane layers is more important that in other systems with higher $\eta_{\mathrm{s}}$. For instance, in figure 2 (a thinner membrane yielding $\eta_{\mathrm{s}}=$ 0.1961 ) or in figure 3 (a thinner electrolyte layer yielding $\eta_{\mathrm{s}}=0.1549$ ), a lesser influence of the lower layers to the radial diffusion is seen; whereas in figure 4 (a more impermeable membrane, $\eta_{\mathrm{s}}=0.0177$ ) the radial diffusion within the ensemble electrolyte and membrane layer is increased. 
Parenthetically, we see in figures 1-4 that the behaviour of the flux lines in the membrane is practically of planar diffusion (flux lines almost in the $\mathrm{z}$ direction, due to the "refraction" when entering the membrane which has been taken as a less permeable medium). Planar diffusion within the membrane coupled with radial diffusion within the electrolyte was the simplifying assumption of Jensen et. al $^{11}$ in the derivation of an approximate expression for the intensitity current. So we can expect a better agreement of the Jensen et al. approach with the rigorous solution for systems with low $\mathrm{z}_{\mathrm{e}}$ (when one-dimensional reduction of the flux within the electrolyte layer is less crude) and with thin and more impermeable membranes (when the flux lines come closer to the normal).

The edge effect indices may also be used in a gas sensor. In this case, the sum of the edge effect indices will be less than 1, because a planar contribution is present. The value of the planar contribution can be numerically computed with the formulae for $\eta_{\mathrm{s}}$ in an infinitely permeable sample (e.g. $\mathrm{P}_{\mathrm{s}} 10^{12}$ times greater than any other permeability).

\section{Parametric study}

\section{Effect of the membrane permeability}

The influence of the membrane permeability on the non-dimensional current (for 3 different membrane thickness) fixing all other parameters, is seen in Fig. 5. Markers $\times$ stand for the thinnest membrane, markers stand for the thickest membrane and the solid line for the one with an intermediate thickness; in all cases, the sample and electrolyte permeabilities are the same $\left(\mathrm{P}_{\mathrm{e}}=\mathrm{P}_{\mathrm{s}}\right)$. For practical representation purposes, the logarithm of the relation $\mathrm{P}_{\mathrm{m}} / \mathrm{P}_{\mathrm{e}}$ is taken to be the abscissa. When all the permeabilities are equal, $\left(\mathrm{P}_{\mathrm{e}}=\mathrm{P}_{\mathrm{m}}=\mathrm{P}_{\mathrm{s}}\right.$ or equivalently $\left.\log \left(\mathrm{P}_{\mathrm{m}} / \mathrm{P}_{\mathrm{e}}\right)=0\right)$ the non-dimensional current becomes unity regardless of the thickness of the membrane, as expected for a single layer according to the definition of the non-dimensional current. If the permeability of the membrane is less than $\mathrm{P}_{\mathrm{e}}=\mathrm{P}_{\mathrm{s}}$ (left of the figure), nondimensional currents less than unity are found, whereas for higher permeability of the membrane (which is not usually available experimentally) non-dimensional currents greater than unity arise as the membrane facilitates the arrival of the electroactive species to the electrode when compared with a single medium.

When $\mathrm{P}_{\mathrm{m}}$ tends to infinity (at the right of figure 5.), the intensity tends asymptotically to a certain value, regardless the membrane thickness. This case could be straightforwardly modelled by imposing bulk partial pressure as boundary conditions along the membraneelectrolyte interface $\left(\mathrm{u}(\mathrm{r}, \mathrm{z})=1, \mathrm{z}=\mathrm{z}_{\mathrm{e}}, \forall \mathrm{r}\right.$ ). This rather unlikely physical case, can, however, be seen as a limit of a gas sample or thoroughly stirred liquid sample with a highly permeable and thin membrane and specific formulas to deal with this case have been discussed elsewhere 20

In fig 5, it can be noticed that the non-dimensional current diminishes indefinitely as the permeability of the membrane decreases. When $\mathrm{P}_{\mathrm{m}}$ tends to 0 , the system approaches a single medium with finite $z$, where steady state is not attainable ${ }^{20}$. Of course, an impermeable membrane is of no use in a sensor, but this limiting case helps in physically understanding that, for any permeabilities and thickness of the layers, the flux crossing a cylindrical surface at $r=\infty$ within a finite-z electrolyte layer is infinitesimal.

Comparison of figures 1 and 4 shows graphically that the decrease in the membrane permeability forces the flux lines to be more compressed within the electrolyte layer, 
enhancing its radial contribution. We also notice a closer approach of the "refracted" flux towards the normal to the interface for higher permeabilities (the phenomenon being due to the imposed equality in the fluxes at the interface as boundary conditions).

\section{Effect of the thickness of the membrane}

Comparing the three thickness in Fig 5 , it is also seen that thicker membranes ( markers) give smaller currents when $\mathrm{P}_{\mathrm{m}}<\mathrm{P}_{\mathrm{e}}$ (as is usually the case). This is to be expected as a low membrane permeability restricts transport of the electroactive species across the membrane. On the same basis, thicker membranes increase the current when $\mathrm{P}_{\mathrm{m}}>\mathrm{P}_{\mathrm{e}}$ as the electroactive species transport is reinforced. In Fig 6 the non-dimensional current is plotted against the thickness of a membrane with $\mathrm{P}_{\mathrm{e}}=\mathrm{P}_{\mathrm{s}}>\mathrm{P}_{\mathrm{m}}$ (this means $\varepsilon_{1}=\varepsilon_{2}<1$ ) and $\mathrm{z}_{\mathrm{e}}$ fixed. If the membrane thickness tends to 0 , the unity non-dimensional current is achieved. Very steep descent is seen next to the origin (corresponding physically to the introduction of a new less permeable layer), while an asymptotic value is tended to for very thick membranes, corresponding to the physical case of two media ${ }^{20}$ : an- electrolyte layer of thickness $z_{\mathrm{e}}$ covered with a semi-infinite membrane space, characterised just by the parameters $z_{\mathrm{e}}$ and $\varepsilon_{1}$.

Comparison of figures 1 and 2 shows that a thinner membrane introduces a smaller distortion on the direction of the flux lines on both sides of it.

\section{Effect of the thickness of the electrolyte layer}

Moving the membrane away from the electrode is considered in figure 7 , where $z_{e}$ and $z_{m}$ change so that the membrane thickness remains constant, i.e., we only increase the height of the electrolyte layer. The plot depicts a growing function, as physically expected from a more important contribution of the electrolyte layer (which in this case is more permeable than the membrane). When the membrane is sufficiently distant, the asymptotic value of 1 (corresponding to 1 medium) is attained.

Moreover, further calculations confirm the physically expected result that the current obtained with a thicker electrolyte layer is more insensible to the thickness and permeability of the membrane or to the permeability of the sample than the current obtained with a thinner electrolyte layer.

At the other extreme, for $\mathrm{z}_{\mathrm{e}}$ tending to 0 , the current tends to a non-zero value. With the parameters of Fig 7 we find a limiting non-dimensional current of 0.0943 for a very thin electrolyte layer given by $z_{\mathrm{e}}=0.001$. If we consider the limit of an infinitely thin electrolyte layer, the system could be described just by a two-layer model ${ }^{20}$ (i.e. membrane and sample), with which one obtains 0.0890 (once we have re-normalised to the appropriate parameter values) that can be seen as the limit value approached by the previous three-layer value of 0.0943. Physically, this means continuity in the dimensional current when the electrolyte layer disappears.

According to the model here assumed, the electrolyte layer extends out to infinity in the rdirection, which in practical terms may require an insulator radius up to 100 times the electrode radius. Some practical design of membrane covered sensors may not be reasonably described by the present model ${ }^{30}$. For instance, some systems could present experimental steady-state currents decreasing with the increase of electrolyte layer thickness ${ }^{31}$. This would be the case if the electrolyte layer does not extend very far relative to the size of the electrode; then the measured steady-state current corresponds to an electroactive species depletion that extends to all the finite volume.

Graphically, we can see the effect of moving the membrane closer to the electrode by comparing figures 1 and 3 . On one hand, the flux lines within the electrolyte layer are 
compressed, but some of them are forced to cross the membrane at smaller $r$. The overall effect, in this case, is a decrease in the $\eta_{\mathrm{e}}$ and an increase in $\eta_{\mathrm{s}}$.

\section{Electrode radius effect}

In our formalism the electrode radius is taken as the unity of length and its actual value does not appear in the expressions. Changing the disc size, while fixing all other dimensional magnitudes, may be achieved by simultaneously changing the non-dimensional distances $Z_{m}$ and $\mathrm{z}_{\mathrm{e}}$ keeping their proportion $\mathrm{w}=\mathrm{z}_{\mathrm{m}} / \mathrm{z}_{\mathrm{e}}$ unaltered. In figure 8 , the non-dimensional current for a system where only the radius is changing is plotted with a continuous line. A striking feature is the existence of a minimum, which disappears if the dimensional current (which can be obtained by multiplying the non-dimensional current by $i_{d, e}$ as given in expression (2) ) is plotted against the radius: then an almost linear increase in the dimensional current follows an increase of the electrode radius. In figure 8 a limiting value of 1 is tended to for radius tending to 0 , this is due to the practical achievement of the steady state within the electrolyte layer, as the fixed membrane is becoming infinitely far away relative to a vanishing electrode radius. Also the unity value is expected for the non-dimensional current when $r_{c} \rightarrow \infty$, as the fixed thickness of the membrane ( $5 \mu \mathrm{m})$ is progressively seen as vanishing, and the systems tends to a single medium where sample and electrolyte merge $\left(\mathrm{P}_{\mathrm{e}}=\mathrm{P}_{\mathrm{s}}\right)$. In the case of a gas sample, the non-dimensional current would increase indefinitely when its radius becomes infinite, as the relative distance between the electrode and points with bulk pressure value vanishes.

\section{Approximated Expressions}

We have described elsewhere ${ }^{20}$ that if there is no need for highly accurate results, then numerical integration may be avoided by using approximate analytical expressions (which are also valid in liquid samples) for the current. These expressions may also be used in estimating an unknown permeability. A particularly convenient formula is:

$$
\frac{\mathrm{i}}{\mathrm{i}_{\mathrm{d}, \mathrm{e}}} \cong \frac{1}{1+2\left[\varepsilon_{1} \mathrm{j}_{0,0}\left(2 \mathrm{z}_{\mathrm{e}}\right)-\varepsilon_{2} \mathrm{j}_{0,0}\left(2 \mathrm{z}_{\mathrm{m}}\right)+\varepsilon_{1} \mathrm{k}_{1} \mathrm{e}^{2 \mathrm{k}_{2} \lambda_{1}} \mathrm{j}_{0,0}\left(2\left(\mathrm{z}_{\mathrm{e}}+\mathrm{k}_{2}\right)\right)-\varepsilon_{2} \mathrm{k}_{1} \mathrm{e}^{2 \mathrm{k}_{2} \lambda_{1}} \mathrm{j}_{0,0}\left(2\left(\mathrm{z}_{\mathrm{m}}+\mathrm{k}_{2}\right)\right)\right]}
$$

where

$$
\lambda_{1}=\frac{1}{2\left(\mathrm{z}_{\mathrm{m}}-\mathrm{z}_{\mathrm{e}}\right)} \ln \left(\frac{\varepsilon_{2} \mathrm{z}_{\mathrm{m}}}{\varepsilon_{1} \mathrm{z}_{\mathrm{e}}}\right) \quad \lambda_{2}=\frac{1}{2\left(\mathrm{z}_{\mathrm{m}}-\mathrm{z}_{\mathrm{e}}\right)} \ln \left(\frac{\varepsilon_{2} \mathrm{z}_{\mathrm{m}}^{2}}{\varepsilon_{1} \mathrm{z}_{\mathrm{e}}^{2}}\right)
$$

and

$$
\mathrm{j}_{0,0}(\mathrm{x})=\frac{2}{\pi} \operatorname{ArcTan}\left(\frac{2}{\mathrm{x}}\right)-\frac{\mathrm{x}}{2 \pi} \ln \left(1+\frac{4}{\mathrm{x}^{2}}\right)
$$

It can be seen in figure 8 ( markers) that, for these parameters, the approximate expression (13) is very accurate.

In the range $\mathrm{z}_{\mathrm{e}}<\mathrm{r}_{\mathrm{c}}$, we have also suggested ${ }^{20}$ the suitability of an even simpler approximation. For $\mathrm{z}_{\mathrm{e}}$ high enough and $\varepsilon_{1}, \varepsilon_{2}$ small enough, the current can be approximated by 
$\frac{\mathrm{i}}{\mathrm{i}_{\mathrm{d}, \mathrm{e}}} \approx 1-\frac{\mathrm{L}_{0,0}^{(1)}}{\mathrm{z}_{\mathrm{e}}}+\left(\frac{\mathrm{L}_{0,0}^{(1)}}{\mathrm{z}_{\mathrm{e}}}\right)^{2}-\frac{\left(\mathrm{L}_{0,0}^{(1)}\right)^{3}+\mathrm{L}_{0,0}^{(3)}}{\mathrm{z}_{\mathrm{e}}^{3}}$

where

$$
\begin{aligned}
& \mathrm{L}_{0,0}^{(1)}=\frac{2}{\pi}\left[\frac{\varepsilon_{2}\left(\varepsilon_{1}^{2}-1\right)}{\mathrm{w}-1} \Phi\left(\varepsilon_{1} \varepsilon_{2}, 1, \frac{\mathrm{w}}{\mathrm{w}-1}\right)+\varepsilon_{1}+\varepsilon_{1}^{2} \Phi\left(\varepsilon_{1}, 1,2\right)-\varepsilon_{1} \varepsilon_{2} \Phi\left(\varepsilon_{1}, 1, \mathrm{w}+1\right)\right] \\
& \mathrm{L}_{0,0}^{(3)}=\frac{-1}{3 \pi}\left[\frac{\varepsilon_{2}\left(\varepsilon_{1}^{2}-1\right)}{(\mathrm{w}-1)^{3}} \Phi\left(\varepsilon_{1} \varepsilon_{2}, 3, \frac{\mathrm{w}}{\mathrm{w}-1}\right)+\varepsilon_{1}+\varepsilon_{1}^{2} \Phi\left(\varepsilon_{1}, 3,2\right)-\varepsilon_{1} \varepsilon_{2} \Phi\left(\varepsilon_{1}, 3, \mathrm{w}+1\right)\right]
\end{aligned}
$$

and $\Phi$ is the Lerch Phi function. A sample of Mathematica code for this approximation is given in Appendix B. The intensity current computed with (16) is depicted with markers o in figure 8 . It is seen that the approximation converges to the exact non-dimensional current when the radius tends to 0 , i.e. when the electrolyte layer becomes much greater than the electrode radius, both attaining the limiting value of 1 for $r=0$. As expected, the approximation becomes useless when $z_{e}$ approaches $r_{c}$. A particular use of (16) consists in estimating the current corresponding to any radius, once we know the current for 2 (preferably extreme) values of the radius, as explained elsewhere ${ }^{20}$.

\section{Computation of permeabilities}

The knowledge of the functional dependence of the non-dimensional current from the geometrical $\left(\mathrm{z}_{\mathrm{e}}\right.$ and $\left.\mathrm{z}_{\mathrm{m}}\right)$ and diffusional $\left(\mathrm{P}_{\mathrm{m}}, \mathrm{P}_{\mathrm{e}}, \mathrm{P}_{\mathrm{s}}\right)$ parameters gives way to a number of fitting procedures that allow the determination of the unknown permeabilities. The most rigorous procedures would involve solving the truncated linear system (to a large enough size) with accurate evaluation of the coefficients via (5). However, approximate expressions ${ }^{20}$ can help in finding the unknown parameter to within the experimental precision much more quickly and easily.

Thus, as an example, we will deal here with an approximate procedure for a very thin electrolyte layer $\left(\mathrm{z}_{\mathrm{m}}>>\mathrm{z}_{\mathrm{e}}\right.$ and $\left.\mathrm{z}_{\mathrm{e}}<<\mathrm{r}_{\mathrm{c}}=1\right)$. Under that restriction (13) becomes

$$
\frac{\mathrm{i}}{\mathrm{i}_{\mathrm{d}, \mathrm{e}}} \cong \frac{1}{1+\mathrm{L}_{0,0}} \cong \frac{1}{1+\frac{2}{1+\frac{\mathrm{z}_{\mathrm{e}}}{\mathrm{z}_{\mathrm{m}}}-\varepsilon_{1}\left(\frac{\varepsilon_{1} \mathrm{z}_{\mathrm{e}}}{\varepsilon_{2}\left(1-\varepsilon_{1}\right) \mathrm{z}_{\mathrm{m}}}\right)^{\mathrm{z}_{\mathrm{e}} / \mathrm{z}_{\mathrm{m}}}}\left\{\varepsilon_{1} \mathrm{j}_{0,0}\left(2 \mathrm{z}_{\mathrm{e}}\right)-\varepsilon_{2} \mathrm{j}_{0,0}\left(2 \mathrm{z}_{\mathrm{m}}\right)\right\}}
$$

Algebraic manipulation of (19) with $\mathrm{P}_{\mathrm{e}}=\mathrm{P}_{\mathrm{s}}$ yields

$$
\varepsilon=\varepsilon_{1}=\varepsilon_{2}=\frac{\left(\frac{i_{d, e}}{i}-1\right)\left(1+\frac{z_{e}}{z_{m}}\right)}{2\left[j_{0,0}\left(2 z_{e}\right)-j_{0,0}\left(2 z_{m}\right)\right]+\left(\frac{i_{d, e}}{i}-1\right)\left(\frac{z_{e}}{z_{m}(1-\varepsilon)}\right)^{z_{e} / z_{m}}}
$$

which allows us to calculate $\varepsilon$ using an iterative procedure. For instance, if we take the known (experimental) non-dimensional current to be 0.12 (in fact, computed through the rigorous procedure with $\varepsilon=0.9214, \mathrm{z}_{\mathrm{e}}=9.01$ and $\mathrm{z}_{\mathrm{m}}=0.51$ ), introducing a seed of 0.9000 in $(20)$, it 
finally converges to $\varepsilon=0.9154$, which is only $0.5 \%$ error from the exact value. It is noticeable that the same procedure fails to converge if $\mathrm{z}_{\mathrm{e}}=0.1$ and $\mathrm{z}_{\mathrm{m}}=0.6$ as expected from the condition $\mathrm{z}_{\mathrm{e}}<<\mathrm{z}_{\mathrm{m}}$ being not fulfilled.

\section{Conclusions}

The easy implementation of the solution of the dual integral equation describing steady state at an inlaid disk electrode covered with a multilayered media (extending out to infinity) allows fast computation of the intensity current. Using this new method we have been able to demonstrate in this paper the particular functional dependence of the intensity current on the physical parameters (i.e. thickness and permeability of each medium). We have shown that the edge-effect produces a current which is dependent, to a very important extent, on the radial component within the electrolyte (and membrane) layer(s), although the steady state requires that all the flux lines have their origin in the sample layer. Definition and formulae for the edge effect indices of each layer allow a fast characterisation of the physical interaction of the parameters involved (thickness and permeabilities).

More accurate permeability determinations now seem feasible with the use of either the exact or the approximate expressions corresponding to the axisymmetric model, overcoming the need to rely on less accurate one-dimensional formulations of the problem.

\section{Acknowledgements}

Financial support from the Spanish Ministry of Education and Science (DGICYT project PB93-0641) is gratefully acknowledged, as well as a grant from the Generalitat de Catalunya (CIRIT, BEAI95) to J. Galceran and from the Wellcome Trust to D. Gavaghan (grant number 038286/Z/93/A). 


\section{Appendix A: Mathematical formulation}

Steady state implies fulfilment of the Laplace equation for the normalised pressure $u$ within each layer. In cylindrical co-ordinates it reads

$$
\frac{\partial^{2} u}{\partial r^{2}}+\frac{1}{r} \frac{\partial u}{\partial r}+\frac{\partial^{2} u}{\partial z^{2}}=0
$$

The boundary conditions ${ }^{6,10}$ for diffusion limited current are:

$$
\begin{array}{lll}
\mathrm{z}=0 & \mathrm{r} \leq 1 & \mathrm{u}=0 \\
\mathrm{z}=0 & \mathrm{r}>1 & \frac{\partial \mathrm{u}}{\partial \mathrm{z}}=0 \\
\mathrm{r}=\infty & \forall \mathrm{z} & \mathrm{u}=1 \\
\mathrm{z}=\mathrm{z}_{\mathrm{e}} & \forall \mathrm{r} & \mathrm{u}_{\mathrm{z}=\mathrm{z}_{\mathrm{e}}^{-}}=\mathrm{u}_{\mathrm{z}=\mathrm{z}_{\mathrm{e}}^{+}} \\
\mathrm{z}=\mathrm{z}_{\mathrm{e}} & \forall \mathrm{r} & \mathrm{P}_{\mathrm{e}}\left(\frac{\partial \mathrm{u}}{\partial \mathrm{z}}\right)_{\mathrm{z}=\mathrm{z}_{\mathrm{e}}^{-}}=\mathrm{P}_{\mathrm{m}}\left(\frac{\partial \mathrm{u}}{\partial \mathrm{z}}\right)_{\mathrm{z}=\mathrm{z}_{\mathrm{e}}^{+}} \\
\mathrm{z}=\mathrm{z}_{\mathrm{m}} & \forall \mathrm{r} & \mathrm{u}_{\mathrm{z}=\mathrm{z}_{\mathrm{m}}^{-}}=\mathrm{u}_{\mathrm{z}=\mathrm{z}_{\mathrm{m}}^{+}} \\
\mathrm{z}=\mathrm{z}_{\mathrm{m}} & \forall \mathrm{r} & \mathrm{P}_{\mathrm{m}}\left(\frac{\partial \mathrm{u}}{\partial \mathrm{z}}\right)_{\mathrm{z}=\mathrm{z}_{\mathrm{m}}^{-}}=\mathrm{P}_{\mathrm{s}}\left(\frac{\partial \mathrm{u}}{\partial \mathrm{z}}\right)_{\mathrm{z}=\mathrm{z}_{\mathrm{m}}^{+}} \\
\mathrm{z}=\infty & \forall \mathrm{r} & \mathrm{u}=1
\end{array}
$$




\section{Appendix B: Mathematica Code}

a) Exact solution of (4) (for parameters leading to $f_{3}$ not too flat):

**definitions:

f3[lam_]:=N[(e1 Exp[-2 lam ze] - e2 $\operatorname{Exp[-2~lam~zm])~/~(1-~e1~e2~} \operatorname{Exp[2~lam~(ze-zm)]-~e1~}$ $\operatorname{Exp}[-2$ lam ze] + e2 $\operatorname{Exp}[-2$ lam zm])]

integ[m_n_]:=2 (4 n+1)NIntegrate[1/lam*f3[lam] BesselJ [2m+1/2,lam]

BesselJ[2n+1/2,lam], \{lam,0,upperlim $\}]$

** introduction of a particular set of data:

upperlim $=10 ; \operatorname{mnmax}=5$

pe=2.69 10^-9; pm=1.1 10^-10; ps=2.69 10^-9; e1 $=($ pe-pm $) /(p e+p m) ; e 2=(p s-p m) /(p s+p m)$

$\mathrm{ze}=0.5 ; \mathrm{zm}=1$

** execution:

$\operatorname{Do}[\mathrm{L}[\mathrm{m}, \mathrm{n}]=\operatorname{integ}[\mathrm{m}, \mathrm{n}],\{\mathrm{m}, 0, \operatorname{mnmax}\},\{\mathrm{n}, 0, \mathrm{~m}\}] ; \mathrm{Do}[\mathrm{L}[\mathrm{m}, \mathrm{n}]=(4 \mathrm{n}+1) /(4 \mathrm{~m}+1) \mathrm{L}[\mathrm{n}, \mathrm{m}],\{\mathrm{m}, 0, \mathrm{mnm}$ ax $\},\{\mathrm{n}, \mathrm{m}+1, \operatorname{mnmax}\}]$

LinearSolve[Transpose[Table[L[i,j], \{i,0,mnmax $\},\{\mathrm{j}, 0, \operatorname{mnmax}\}]$

+ IdentityMatrix[mnmax +1$]]$, Table[If[i>0,0,N[Sqrt[2/Pi]]],

$\{\mathrm{i}, 0, \operatorname{mnmax}\}]][[1]] \mathrm{Sqrt}[\mathrm{Pi} / 2] / / \mathrm{N}$

** obtained non-dimensional current value:

Out $[25]=0.514062$

b) Approximated expression (16) for high $\mathrm{z}_{\mathrm{e}}$ :

$* *$ definition of functions:

L00f[e1_,e2_,w_] := 2/Pi*(e2 (e1^2-1)/(w-1) LerchPhi[e1 e2,1,w/(w-1)] + e1 + e1^2

LerchPhi[e1,1,2]-e1 e2 LerchPhi[e1,1,w+1])

L00t[e1_,e2_, w_] :=-1/(3Pi)*( e2 (e1^2-1)/(w-1)^3 LerchPhi[e1 e2,3,w/(w-1)] + e1 + e1^2

LerchPhi[e1,3,2]-e1 e2 LerchPhi[e1,3,w+1])

Intens[e1_,e2_,ze_zm_]:=1 - L00f[e1,e2,zm/ze]/ze -L00t[e1,e2,zm/ze]/ze`3

$+(\text { L00f }[\mathrm{e} 1, \mathrm{e} 2, \mathrm{zm} / \mathrm{ze}] / \mathrm{ze})^{\wedge} 2-(\mathrm{L} 00 \mathrm{f}[\mathrm{e} 1, \mathrm{e} 2, \mathrm{zm} / \mathrm{ze}] / \mathrm{ze})^{\wedge} 3$

** example of execution

$\mathrm{N}[$ Intens$[0.9214,0.9214,2,4]]$ Out[14] $=0.754935$ 


\section{Appendix C: Symbols}

\begin{tabular}{|c|c|c|}
\hline Symbol & Meaning & \begin{tabular}{|l} 
Eqn. \\
involved
\end{tabular} \\
\hline$*$ & bulk superscript & $(3)$ \\
\hline $\mathrm{a}_{\mathrm{m}}$ & $\mathrm{m}$-th coefficient of the unknown function expansion & $(4)$ \\
\hline $\mathrm{c}$ & electroactive species concentration & $(3)$ \\
\hline $\mathrm{D}_{\mathrm{e}}$ & $\begin{array}{|llll|}\begin{array}{l}\text { diffusion coefficient of electroactive species in } \\
\text { electrolyte medium }\end{array} & & \\
\end{array}$ & (3) \\
\hline $\mathrm{e}$ & electrolyte layer subscript & (A-2), Fig. 1 \\
\hline $\mathrm{f}_{3}$ & "weight" function in computing $\mathrm{L}_{\mathrm{m}, \mathrm{n}}$ & $(6),(5)$ \\
\hline $\mathrm{F}$ & Faraday constant & $(3)$ \\
\hline $\mathrm{h}_{\mathrm{e}}, \mathrm{h}_{\mathrm{m}}$ & auxiliary function to compute edge effect index $\eta$ & $(12),(10)$ \\
\hline i & dimensional current & $(3)$ \\
\hline$i_{\mathrm{d}, \mathrm{e}}$ & $\begin{array}{l}\text { diffusion limited steady state current for unshielded } \\
\text { electrode in electrolyte medium }\end{array}$ & $(3)$ \\
\hline $\mathrm{j}_{\mathrm{o}, \mathrm{o}}$ & auxiliary function & $(15)$ \\
\hline $\mathrm{J}_{\mathrm{V}}$ & Bessel function of order $v$ & $(5)$ \\
\hline $\mathrm{L}_{\mathrm{m}, \mathrm{n}}$ & coefficient of $\mathrm{a}_{\mathrm{m}}$ in the nth. equation & $(4),(5)$ \\
\hline $\mathrm{m}$ & membrane layer subscript & (A-2), Fig. 1 \\
\hline$n$ & number of exchanged electrons & $(3)$ \\
\hline $\mathrm{p}(\mathrm{r}, \mathrm{z})$ & partial pressure of electroactive species & $(3)$ \\
\hline $\mathrm{P}_{\ell}$ & Permeability of electroactive species in layer $\ell(e, m, s)$ & $(\mathrm{A}-2)$ \\
\hline $\mathrm{r}$ & radial co-ordinate & (A-1), Fig. 1 \\
\hline $\mathrm{r}_{\mathrm{c}}$ & $\begin{array}{l}\text { cathode (or electrode) radius. Distance normalisation } \\
\text { factor }\end{array}$ & Fig. 1 \\
\hline $\mathrm{s}$ & sample layer subscript & (A-2), Fig. 1 \\
\hline $\mathrm{u}$ & non-dimensional partial pressure $\mathrm{p} / \mathrm{p}^{*}$ & $(\mathrm{~A}-1)$ \\
\hline $\mathrm{W}$ & ratio of the thickness of the layers & $(17)$ \\
\hline $\mathrm{z}$ & axial co-ordinate & (A-1), Fig. 1 \\
\hline $\mathrm{z}_{\mathrm{e}}$ & $\begin{array}{l}\text { distance from cathode to the electrolyte-membrane } \\
\text { interface normalised with respect to the cathode radius } r_{c}\end{array}$ & Fig. 1 \\
\hline $\mathrm{z}_{\mathrm{m}}$ & $\begin{array}{l}\text { distance from cathode to the membrane-sample interface } \\
\text { normalised with respect to the cathode radius } r_{c}\end{array}$ & Fig. 1 \\
\hline$\varepsilon_{1}, \varepsilon_{2}$ & $\begin{array}{l}\text { non-dimensional ratio of permeabilities } \\
\text { (e1 and } 22 \text { in Mathematica code) }\end{array}$ & $(1)$ \\
\hline$\eta_{e}$ & edge effect index of layer $\ell(e, m, s)$ & $(7)$ \\
\hline$\Phi$ & $\Phi(\mathrm{x}, \mathrm{s}, \mathrm{v})=\sum_{\mathrm{n}=0}^{\infty} \frac{\mathrm{x}^{\mathrm{n}}}{(\mathrm{v}+\mathrm{n})^{\mathrm{s}}}$ & $(17)$ \\
\hline
\end{tabular}




\section{References}

1 Clark, L.C., Trans. Am. Soc. Artif. Intern. Organs, 1956, 2, 41.

2 Hahn, C.E.W. , J. Phys. E. Scient. Inst. , 1980, 13, 470.

3 Chang, S.C., Stetter, J.R. and Cha, C.S., Talanta, 1993, 40, 461.

4 Tarnowski, D.J., Bekos, E.J. and Korzeniewski, C., Anal. Chem. , 1995, 67, 1546.

5 Hitchman, M.L., Measurement of Dissolved Oxygen, Wiley, New York, 1978.

6 Myland, J.C. and Oldham, K.B. , J. Electrochem. Soc. , 1984, 131, 1815.

7 Ultman, J.S., Firouztale, E. and Skerpon, M.J., J. Electroanal. Chem. , 1981, 127, 59.

8 Flanagan, J.B. and Marcoux, L. , J. Phys. Chem. , 1973, 77, 1051.

9 Oldham, K.B., J. Electroanal. Chem. , 1981, 122, 1.

10 Gavaghan, D.J., Rollett, J.S. and Hahn, C.E.W. , J. Electroanal. Chem. , 1992, 325, 23.

11 Jensen, O.J., Jacobsen, T. and Thomsen, K. , J. Electroanal. Chem. , 1978, 87, 203.

12 Uhegbu, C.E. and Pardue, H.L., Anal. Chem. , 1992, 64, 2378.

13 Taguchi, K., Yano, S., Hiratani, K. and Minoura, N. , J. Polym. Sci. B Polym. Phys. , 1993, 31, 339.

14 Pyati, R. and Murray, R.W., J. Phys. Chem., 1994, 98, 11129.

15 Taliercio, T., Dilhan, M., Massone, E., Foucaran, A., Gue, A.M., Bretagnon, T., Fraisse, B. and Montes, L., Sensor Actuator. A Phys. , 1995, 46, 43.

16 Hahn, C.E.W., DPhil Thesis, University of Oxford, 1974.

17 Firouztale, E., Skerpon, M.J. and Ultman, J.S., J. Electroanal. Chem. , 1982, 134, 1.

18 Gavaghan, D.J., Rollett, J.S. and Hahn, C.E.W. , J. Electroanal. Chem. , 1993, 348, 1.

19 Galceran, J., Gavaghan, D.J. and Rollett, J.S., J. Electroanal. Chem. , 1995, 394, 17.

20 Galceran, J., Salvador, J., Puy, J., Cecilia, J. and Gavaghan, D.J., submitted to J. Electroanal. Chem. 
21 Tranter, C.J., Quart. Journ. Mech. Appl. Math. , 1950, 3, 411.

22 Tranter, C.J., Integral Transforms in Mathematical Physics, Methuen, London, 1966.

23 Sneddon, I.N., Mixed boundary value problems in potential theory, Wiley, New York, 1966.

24 Wolfram, S., Mathematica. A System for Doing Mathematics by Computer, AddisonWesley Publishing Company, Redwood City (California), 1988.

25 Saito, Y., Rev. Polarogr. Jpn. , 1968, 15, 177.

26 Crank, J., The Mathematics of Diffusion, Oxford University Press, London, 1957.

27 Bond, A.M., Oldham, K.B. and Zoski, C.G. , J. Electroanal. Chem. , 1988, 245, 71.

28 Cope, D.K. and Tallman, D.E. , J. Electroanal. Chem. , 1994, 373, 53.

29 Bond, A.M., Zoski, C.G. and Oldham, K.B., Anal. Chim. Acta , 1989, 216, 177.

30 Fang, Y. and Leddy, J., Anal. Chem. , 1995, 67, 1259.

31 Hale, J.M. , J. Electroanal. Chem. , 1980, 107, 281. 


\section{Figure captions:}

Fig 1 Geometry modelled for the membrane covered inlaid disk electrode. Gradient lines are plotted so that 1/10 of the overall flux of the electroactive species flows inside any "tube" defined by any two adjacent lines. This plot corresponds to $z_{e}=0.5, z_{m}=1$, $P_{e}=P_{s}=2.6910^{-9} \mathrm{~mol} \mathrm{~m}^{-1} \mathrm{~s}^{-1} \mathrm{~atm}^{-1}, P_{m}=1.110^{-10} \mathrm{~mol} \mathrm{~m}^{-1} \mathrm{~s}^{-1} \mathrm{~atm}^{-1}$.

Fig 2 Gradient lines for $z_{e}=0.5, z_{m}=0.6, P_{e}=P_{s}=2.6910^{-9} \mathrm{~mol} \mathrm{~m}^{-1} \mathrm{~s}^{-1} \mathrm{~atm}^{-1}, P_{m}=1.110^{-10}$ mol m $\mathrm{m}^{-1} \mathrm{~atm}^{-1}$. Edge effect indices: $\eta_{s}=0.1961, \eta_{m}=0.0021, \eta_{e}=0.8018$.

Fig 3 Gradient lines for $z_{e}=0.2, z_{m}=0.7, P_{e}=P_{s}=2.6910^{-9} \mathrm{~mol} \mathrm{~m}^{-1} \mathrm{~s}^{-1} \mathrm{~atm}^{-1}, P_{m}=1.110^{-10}$ mol m $\mathrm{m}^{-1} \mathrm{~atm}^{-1}$. Edge effect indices: $\eta_{s}=0.1549, \eta_{m}=0.0267, \eta_{e}=0.8184$.

Fig 4 Flux lines for $z_{e}=0.5, z_{m}=1, P_{e}=P_{s}=2.6910^{-9} \mathrm{~mol} \mathrm{~m}^{-1} \mathrm{~s}^{-1} \mathrm{~atm}^{-1}, P_{m}=1.110^{-11} \mathrm{~mol} \mathrm{~m}^{-}$ ${ }^{1} \mathrm{~s}^{-1} \mathrm{~atm}^{-1}$. Edge effect indices: $\eta_{s}=0.0177, \eta_{m}=0.0012, \eta_{e}=0.9811$.

Fig 5 Effect of the membrane permeability on the non-dimensional current for fixed electrolyte layer thickness $\left(z_{e}=0.5\right)$ and electrolyte and sample permeabilities $\left(P_{e}=P_{s}\right.$ $\left.=2.6910^{-9} \mathrm{~mol} \mathrm{~m}^{-1} \mathrm{~s}^{-1} \mathrm{~atm}^{-1}\right)$. Three thickness are compared: $z_{m}-z_{e}=0.5$ ( $\times$ marker $), z_{m^{-}}$ $z_{e}=2$ (continuous line) and $z_{m}-z_{e}=8$ ( marker).

Fig 6 Effect of changing the thickness of the membrane $\left(\varepsilon_{1}=\varepsilon_{2}=0.9214\right.$, e.g. $P_{e}=P_{s}=2.69$ $\left.10^{-9} \mathrm{~mol} \mathrm{~m}^{-1} \mathrm{~s}^{-1} \mathrm{~atm}^{-1} . \mathrm{Pm}=1.110^{-11} \mathrm{~mol} \mathrm{~m}^{-1} \mathrm{~s}^{-1} \mathrm{~atm}^{-1}\right)$ with a fixed electrolyte layer $\left(z_{e}=\right.$ 2) on the non-dimensional current.

Fig 7 Effect of moving a fixed membrane $\left(z_{m}-z_{e}=0.5, \varepsilon_{1}=\varepsilon_{2}=0.9214\right.$, e.g. $P_{e}=P_{s}=2.69$ $\left.10^{-9} \mathrm{~mol} \mathrm{~m}^{-1} \mathrm{~s}^{-1} \mathrm{~atm}^{-1}, P_{m}=1.110^{-11} \mathrm{~mol} \mathrm{~m}^{-1} \mathrm{~s}^{-1} \mathrm{~atm}^{-1}\right)$ on the non-dimensional current.

Fig 8 Effect of changing the electrode radius (sample-membrane interface height: $15 \mu \mathrm{m}$; membrane-electrolyte interface height: $10 \mu \mathrm{m} ; \varepsilon_{1}=\varepsilon_{2}=0.9214$, e.g. $P_{e}=P_{s}=2.6910^{-9}$ mol m $\left.\mathrm{s}^{-1} \mathrm{~atm}^{-1}, P_{m}=1.110^{-10} \mathrm{~mol} \mathrm{~m}^{-1} \mathrm{~s}^{-1} \mathrm{~atm}^{-1}\right)$ on the non-dimensional current. Markers - stand for the approximate expression (13) and markers o stand for expression (16). 


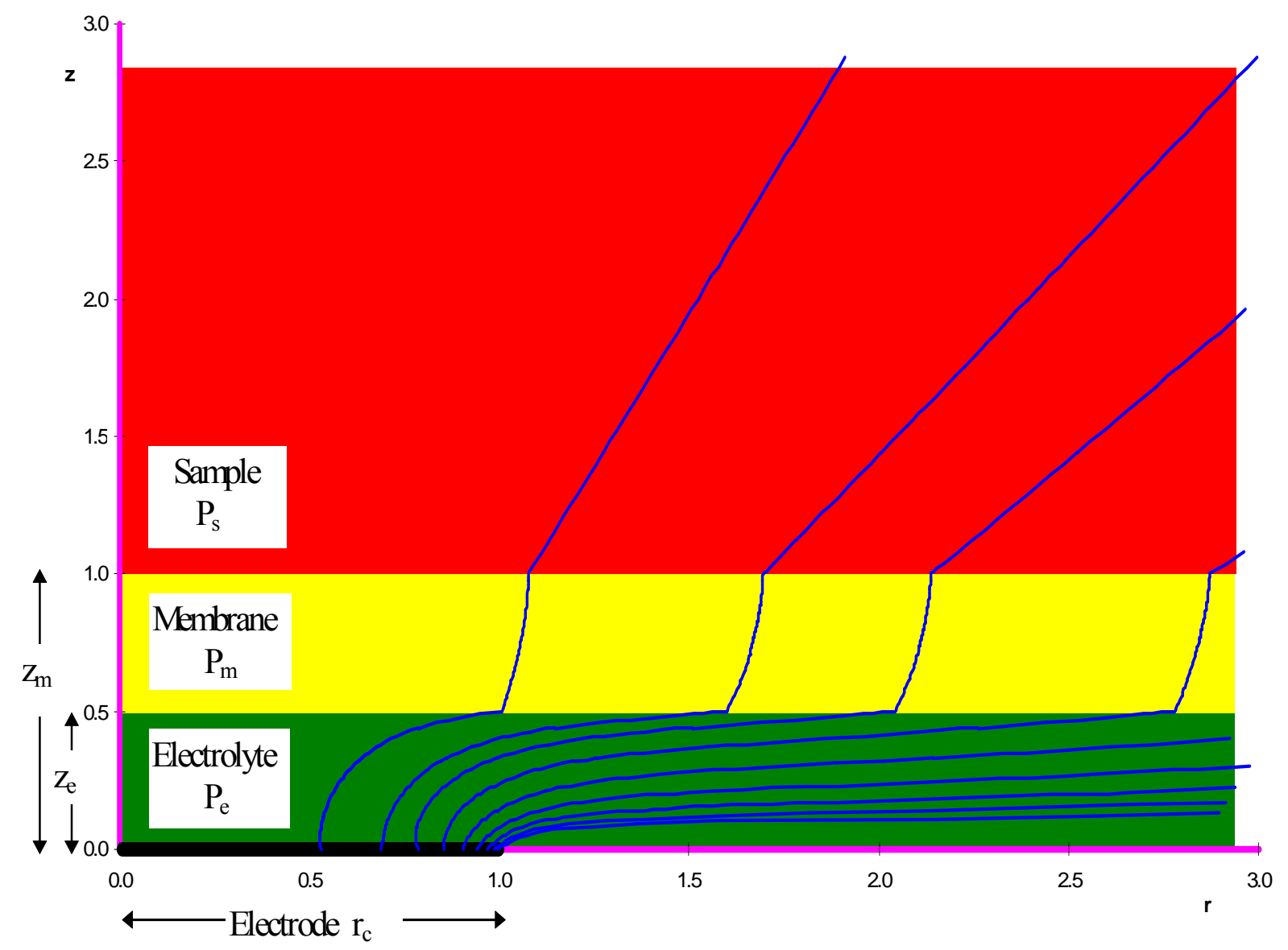

Fig. 1 
Published in Analyst 1996, vol 121, p 1863-1868

DOI: 10.1039/an9962101863

reprints to galceran@quimica.udl.cat

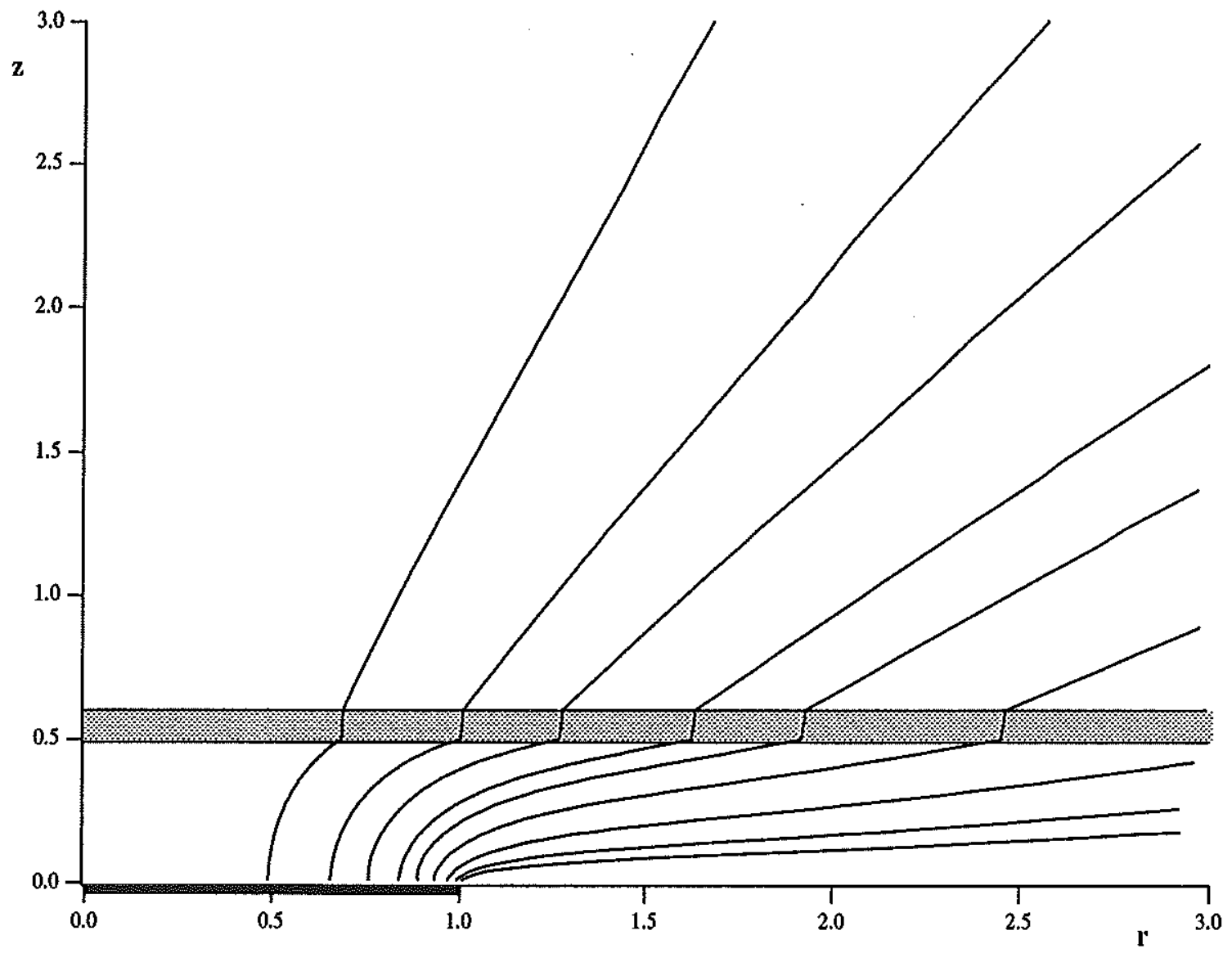

Fig 2 


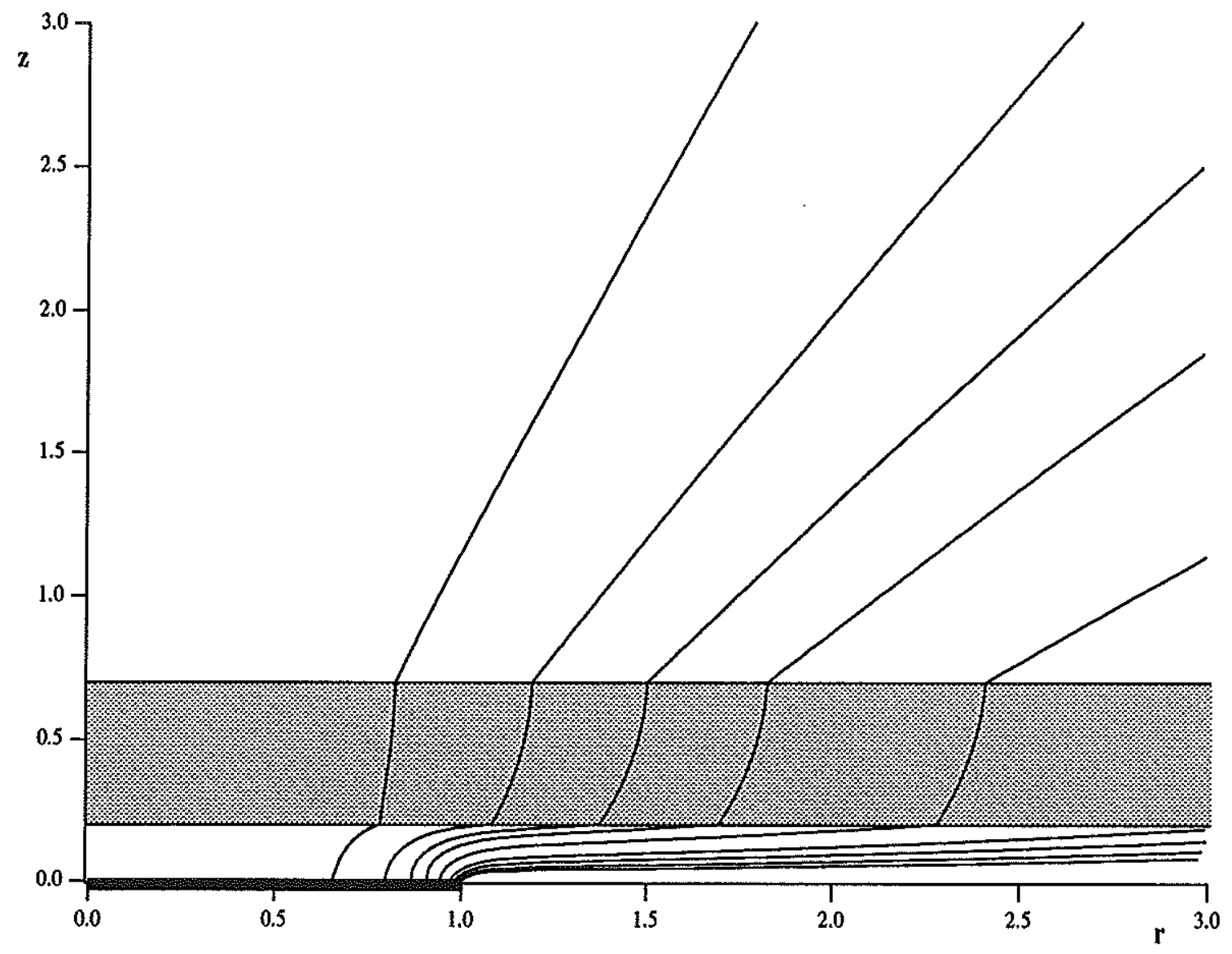

Fig 3 


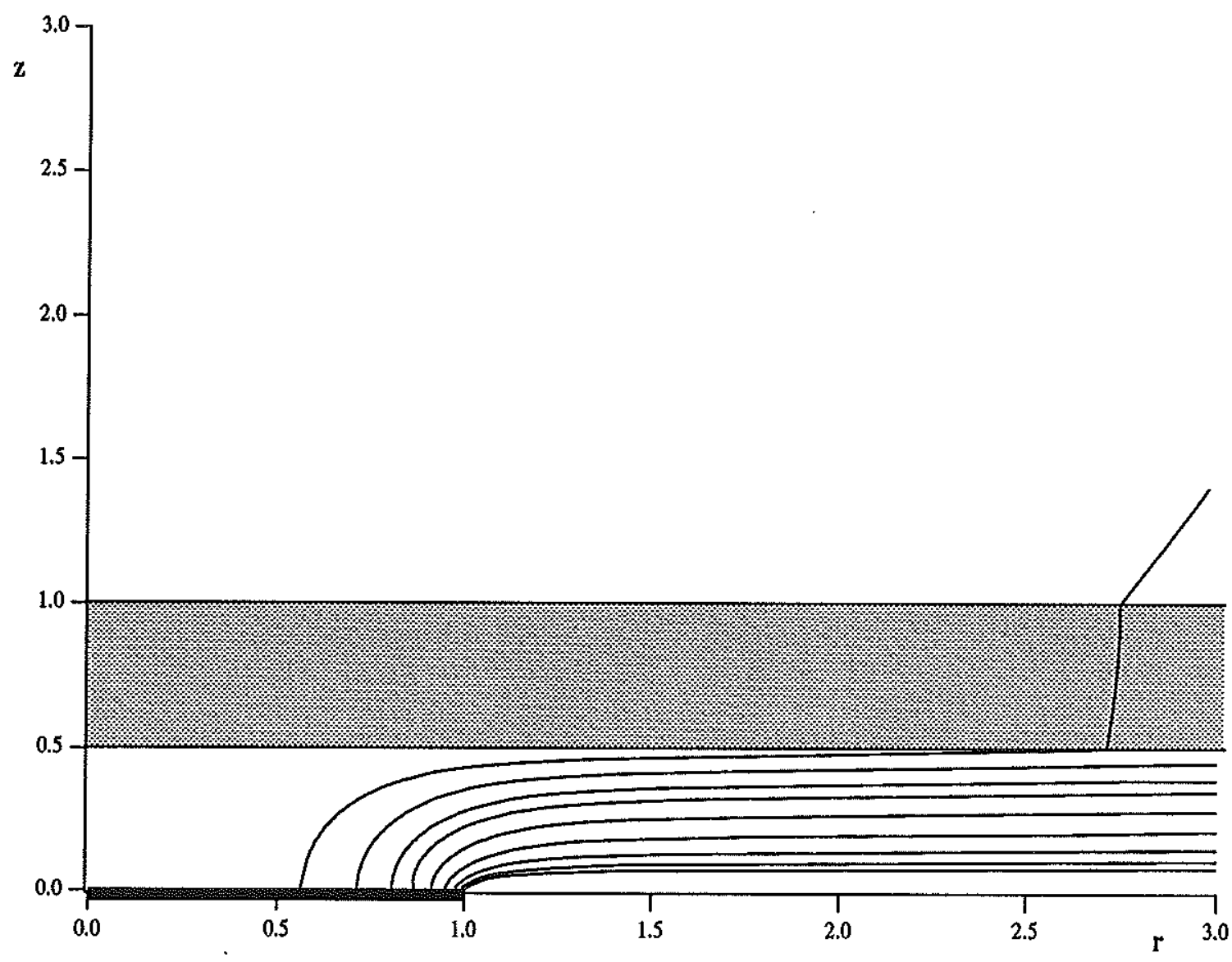




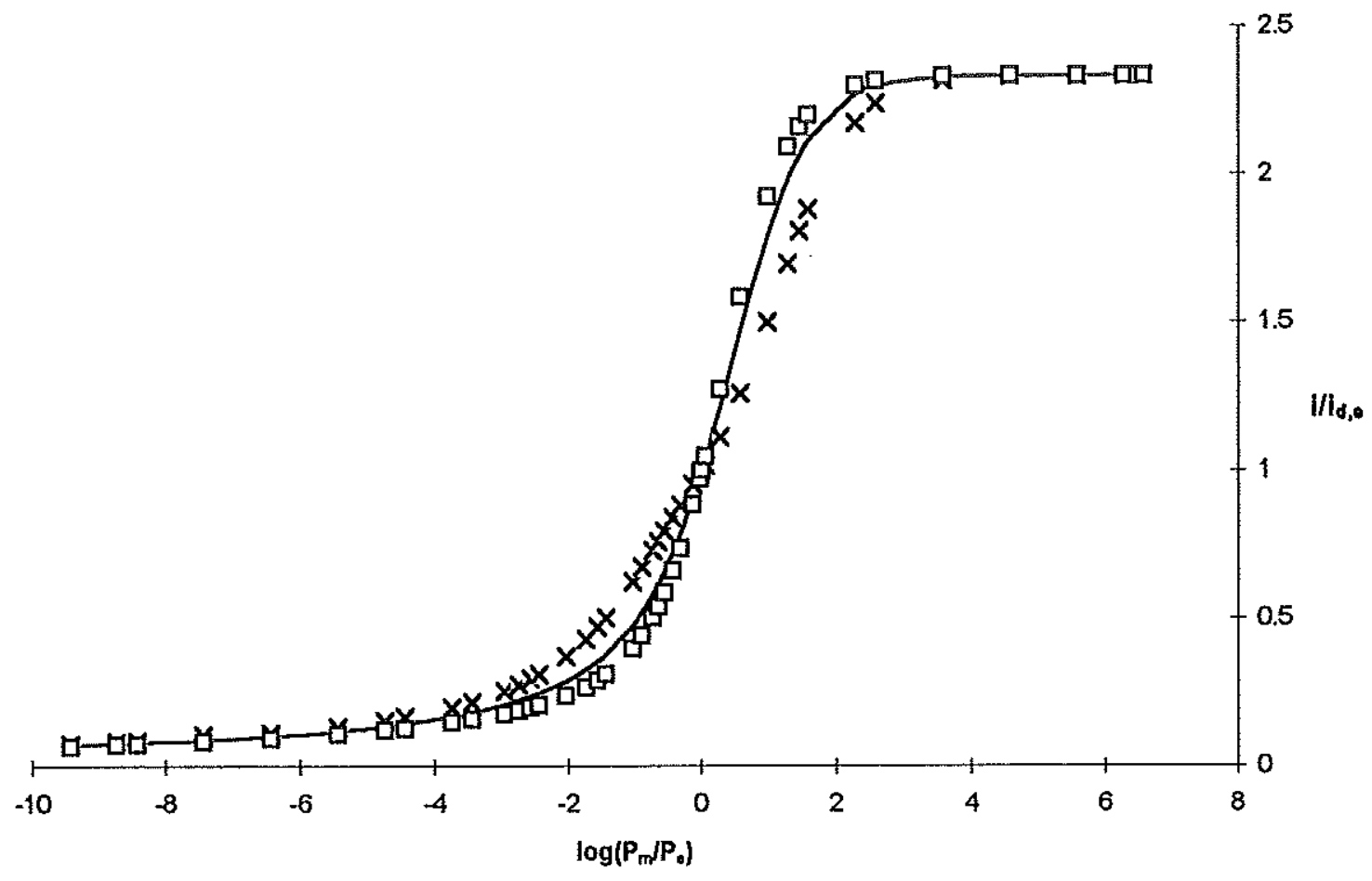

Fig 5 
Published in Analyst 1996, vol 121, p 1863-1868

DOI: 10.1039/an9962101863

reprints to galceran@quimica.udl.cat

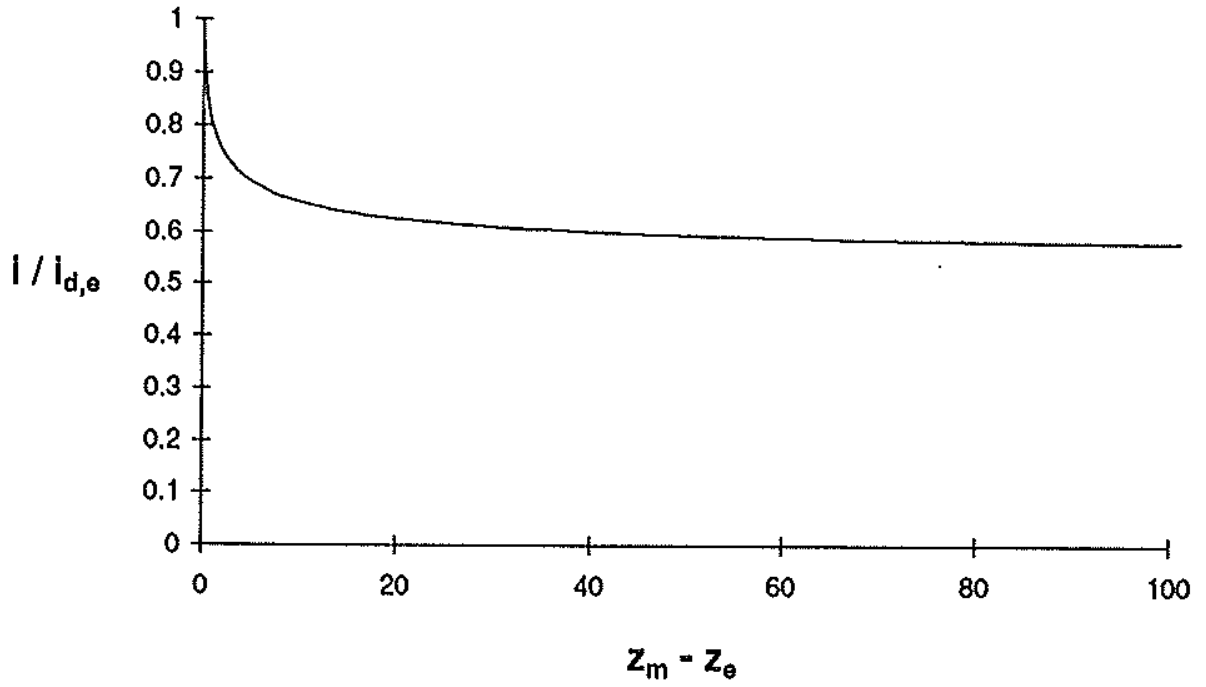

Fig 6 


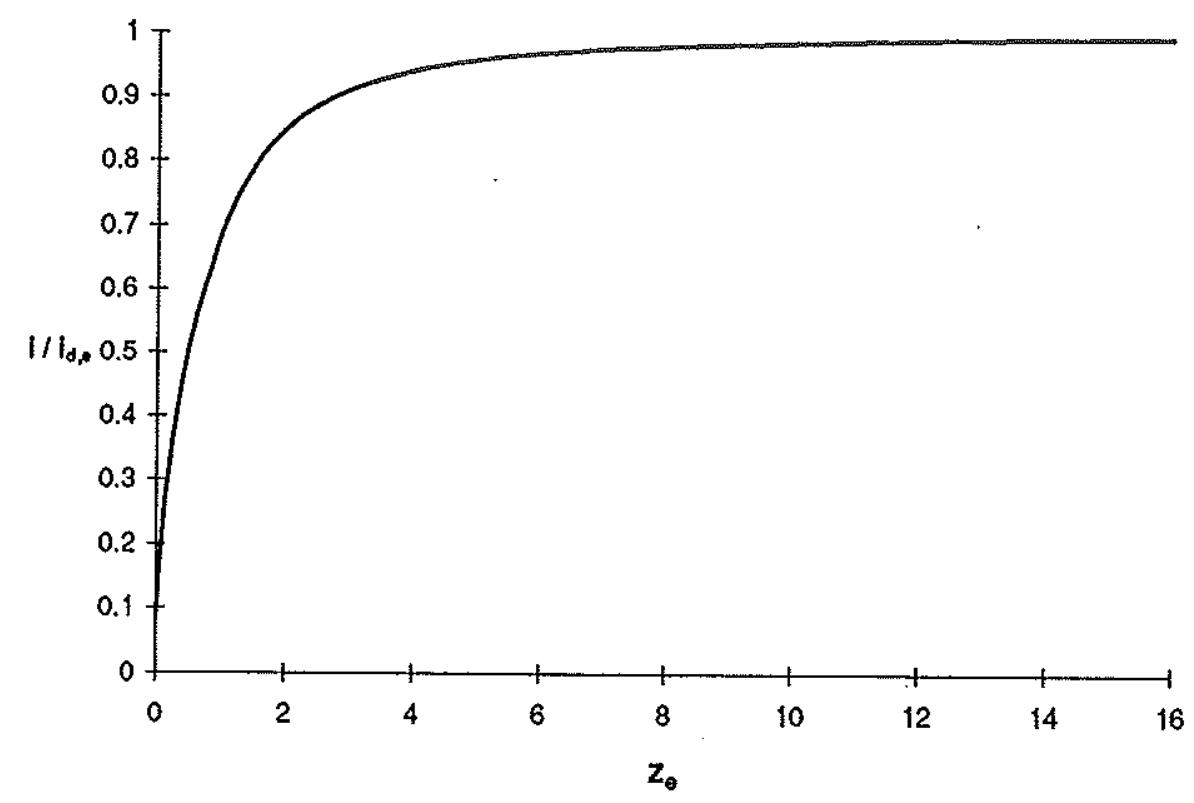

Fig 7 


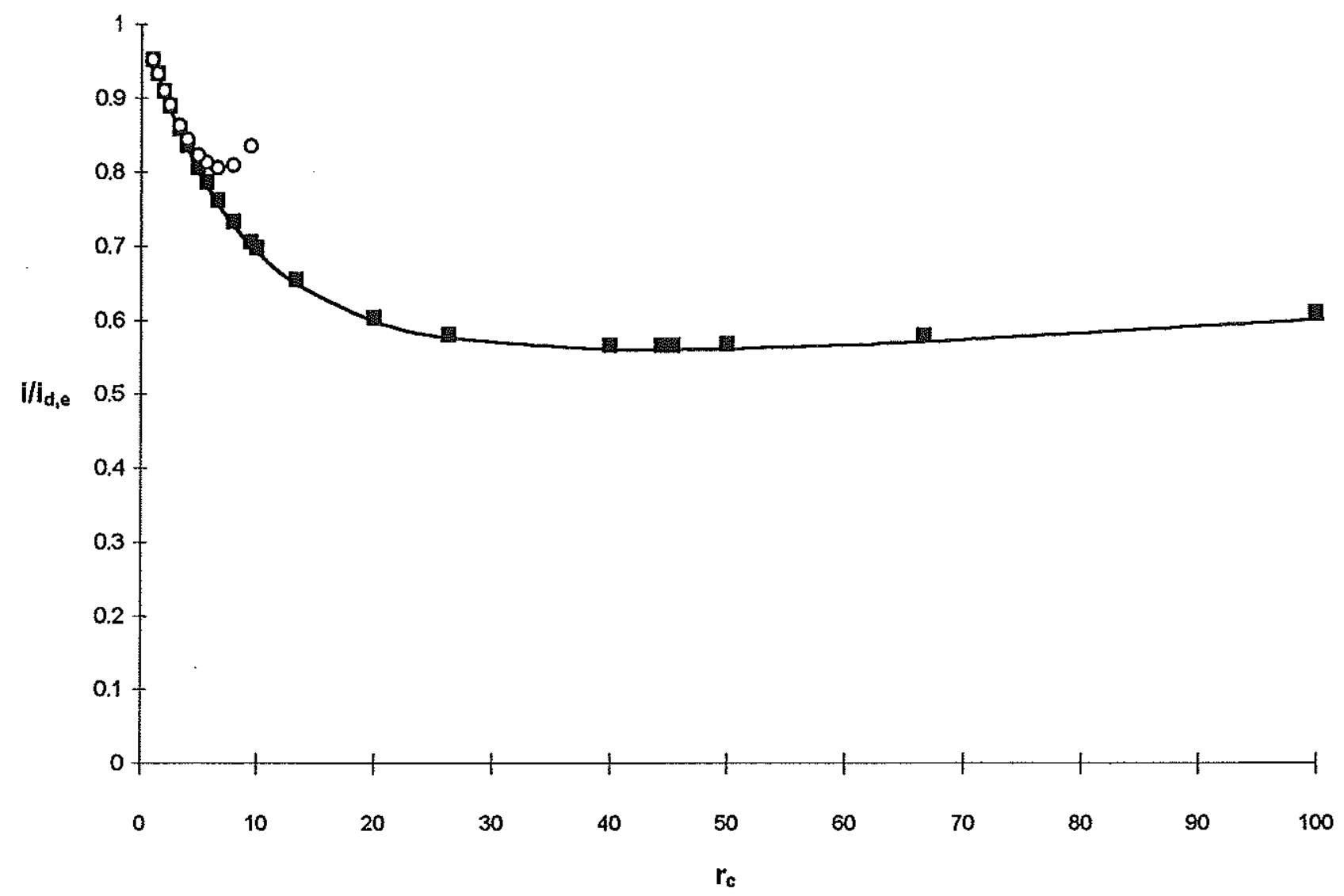

Fig 8 


\section{Edge effect indices: not shown in article Galceran 121 (1996) 1863}

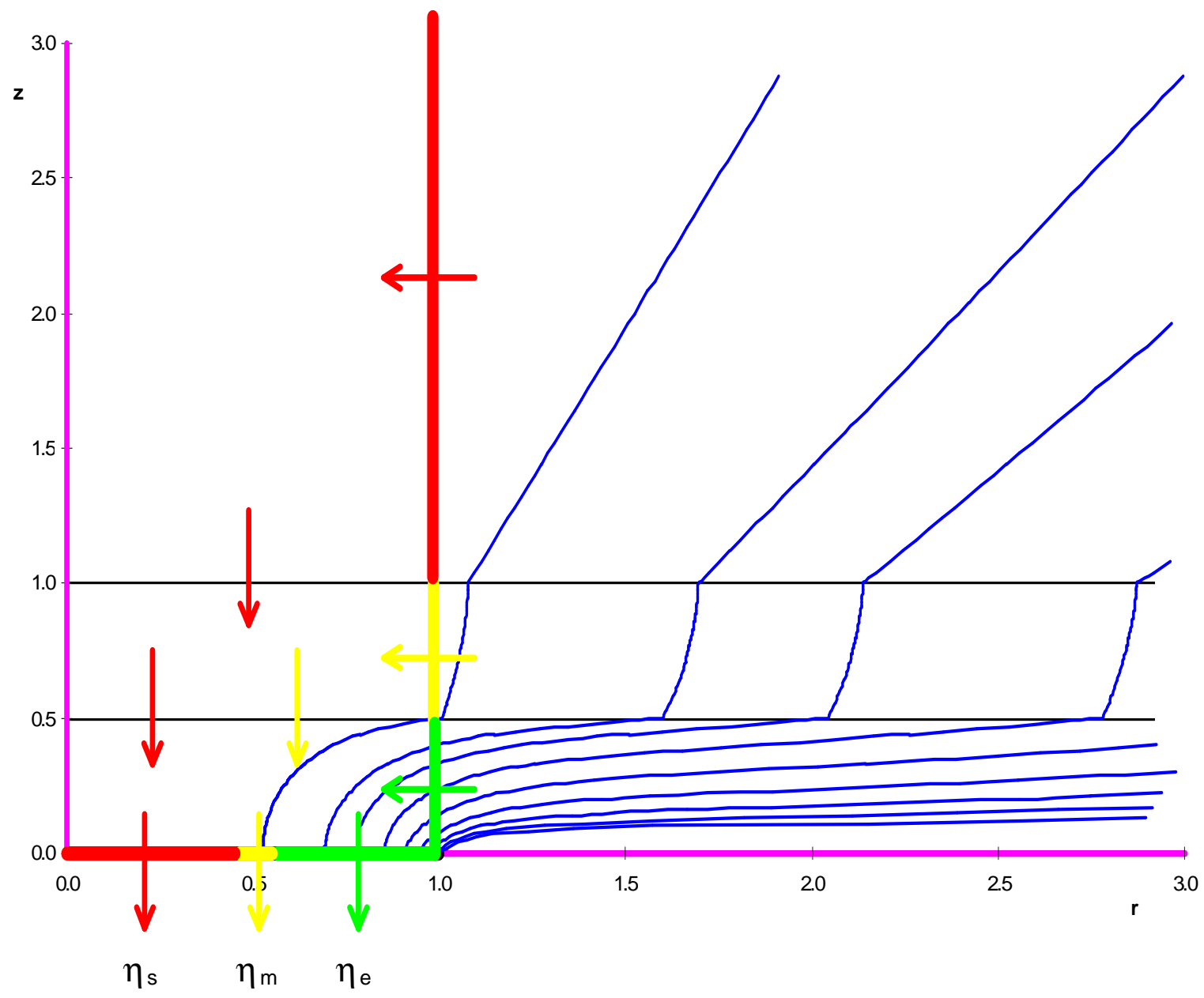

Index of edge effect contribution in the membrane covered inlaid disk electrode. Parameters $z_{e}=0.5, z_{m}=1, P_{e}=P_{s}=2.6910^{-9} \mathrm{~mol} \mathrm{~m}^{-1} \mathrm{~s}^{-1} \mathrm{~atm}^{-1}, P_{m}=1.110^{-10} \mathrm{~mol} \mathrm{~m}^{-1} \mathrm{~s}$ ${ }^{1} \mathrm{~atm}^{-1}$. Edge effect indices: $\eta_{s}=0.0860, \eta_{m}=0.0018, \eta_{e}=0.9032$. 